\title{
Ensemble-Based Targeted Observation Method Applied to Radar Radial Velocity Observations on Idealized Supercell Low-Level Rotation Forecasts: A Proof of Concept
}

\author{
Christopher A. Kerr And Xuguang WAng \\ School of Meteorology, University of Oklahoma, Norman, Oklahoma
}

(Manuscript received 13 June 2019, in final form 25 November 2019)

\begin{abstract}
The potential future installation of a multifunction phased-array radar (MPAR) network will provide capabilities of case-specific adaptive scanning. Knowing the impacts adaptive scanning may have on short-term forecasts will influence scanning strategy decision-making in hopes to produce the most optimal ensemble forecast while also benefiting human severe weather warning decision-making. An ensemble-based targeted observation algorithm is applied to an observing system simulation experiment (OSSE) where the impacts of synthetic idealized supercell radial velocity observations are estimated before the observations are "collected" and assimilated. The forecast metric of interest is the low-level rotation forecast metric $(0-1-\mathrm{km}$ updraft helicity), a surrogate for tornado prediction. It is found that the ensemble-based targeted observation approach can reasonably estimate the true error variance reduction when an effective method that treats sampling error is applied, the period of model forecast is associated with less degrees of nonlinearity, and the observation information content relative to the background forecast is larger. In some scenarios, a subset of a full-volume scan assimilation produces better forecasts than all observations within the full volume. Assimilating the full-volume scan increases the number of potential spurious correlations arising between the forecast metric and radial velocity observation induced state perturbations, which may degrade the forecast metric accuracy.
\end{abstract}

\section{Introduction}

The proposed future installation of a multifunction phased array radar (MPAR) network across the United States will present new opportunities for both the human meteorologist and numerical weather prediction (NWP) models (Weber et al. 2007; Zrnić et al. 2007; Heinselman and Torres 2011; Curtis and Torres 2011; Yussouf and Stensrud 2010; Supinie et al. 2017). The current WSR-88D network requires approximately $4.5 \mathrm{~min}$ to complete a full-volume scan given its mechanically steered beam whereas MPAR will be able to complete a full-volume scan in under $1 \mathrm{~min}$ via electronic beam steering while sharing resources with the Federal Aviation Administration (FAA) for aircraft tracking. This will provide more frequent updates of rapidly evolving convective storms, including tornadic supercells. This will also benefit meteorologists by increasing warning lead times through situational awareness (Heinselman et al. 2012, 2015; Kuster et al. 2015).

Corresponding author: Christopher A. Kerr, christopher.kerr@ noaa.gov
MPAR will have significant impacts on storm-scale NWP and NOAA's Warn-on-Forecast (WoF) program which utilizes very short-term ensemble forecasts to increase warning lead times through radar data assimilation (Stensrud et al. 2009, 2013). Storm-scale radar data assimilation has been applied in numerous studies in recent years (e.g., Snyder and Zhang 2003; Dowell et al. 2004; Tong and Xue 2005; Aksoy et al. 2009; Yussouf and Stensrud 2010, 2012; Yussouf et al. 2013; Wheatley et al. 2014; Johnson et al. 2015; Maejima et al. 2017; Wang and Wang 2017; Maejima et al. 2019). Radar data assimilation produces reliable analyses and subsequent short-term forecasts (up to $3 \mathrm{~h}$ ) of individual convective storms. However, most studies have utilized radar data typical of the WSR-88D (5- to 15-min assimilation cycling) whereas a few have explored frequent updates associated with MPAR (Yussouf and Stensrud 2010; Supinie et al. 2017; Stratman et al. 2020). Yussouf and Stensrud (2010) show through a perfect model observing system simulation experiment (OSSE) that 1-min full-volume updates are able to sufficiently spin up convection over a 15 -min period while conventional 5-min updates require around an hour of assimilation cycling. 
Frequent MPAR data cycling is also beneficial to heterogeneous mesoscale environment experiments and low-level rotation forecasts (Supinie et al. 2017).

Another key feature of MPAR will be both elevation and azimuth adaptive scanning capabilities via electronic beam steering. MPAR will be able to repeatedly scan across a specified azimuth range of interest given meteorological phenomena of interest (e.g., convective storms) rather than the full $360^{\circ}$ range. The other advantage (of particular interest to this study) is the elevation-prioritized scanning strategy (Heinselman and Torres 2011). This technique allows repeated sampling of specified vertical portions of meteorological phenomena without mechanically driven adaptive scanning as in WSR-88D (e.g., low-levels of supercells where tornadogenesis can occur). In scenarios where azimuthal and/or vertical adaptive scanning strategies are utilized combined with possible aviation tracking, full-volume scan observations may not be available.

The allotted operational time designated for weather phenomena observation will have to be used wisely given other potential uses of MPAR (e.g., aircraft tracking). Although a full-volume scan can be completed in $1 \mathrm{~min}$, the adaptive sampling abilities of MPAR described above would provide targeted subsets of a full-volume scan with smaller observation error via increased radar dwell time. Adaptive sampling also has advantages for operational forecasters. There could be rapidly evolving situations (e.g., a tornado impacting a populated area) where subminute low-level observations are beneficial for warning issuance by meteorologists, and a decision will need to be made regarding optimal adaptive scanning to benefit both human forecasters and convective-scale ensemble forecasts while also meeting the needs for aviation tracking. In such described cases, only a subset of a full-volume scan could be available for assimilation into an ensemble guidance system. Given the scenarios where only an observation subset would be available, the potential impacts of targeted subsets versus full-volume scans need to be known before observations are collected. Targeted radar observations may have broad benefits for convective-scale data assimilation and forecasting as well as decision-making and social impacts.

The idea of determining ahead of time how to deploy the observation network before the observations are made is termed as "targeted observations" or "adaptive sampling" (e.g., Palmer et al. 1998; Berliner et al. 1999; Baker and Daley 2000; Bishop et al. 2001; Ancell and Hakim 2007; Torn and Hakim 2008). Targeted observation techniques in past studies have included both adjoint-sensitivity (Buizza and Montani 1999; Gelaro et al. 1999; Langland et al. 1999) and ensemble-based methods (e.g., Bishop et al. 2001; Majumdar et al. 2001; Hamill and Snyder 2002; Torn 2014). Ancell and Hakim (2007) demonstrate the two methods produce different results both in spatial scale of sensitivity fields and order of magnitude. Adjointsensitivity uses only a single adjoint model integration to determine forecast sensitivities. The ensemble-based technique instead uses ensemble forecast and data assimilation equations to estimate or predict the forecast error variance reduction if a set of observations were assimilated (Bishop et al. 2001; Ancell and Hakim 2007; Torn and Hakim 2008; Torn and Hakim 2009). A specific metric to measure variance reduction can be selected based on the user's interest. The method also involves using ensemble regression or ensemble correlation at different times. Previous targeted studies have focused on synoptic-scale and tropical systems (e.g., Majumdar et al. 2001; Torn and Hakim 2008; Torn and Hakim 2009; Torn 2014). Few studies have attempted to utilize targeted observations to improve short-term convective-scale forecasts (e.g., Limpert and Houston 2018). Chang (2014) highlights the importance of assimilating targeted radial velocity in space where background error covariances are significant to improve vertical velocity forecasts. This study applies an ensemble-based targeted observation method to radial velocity observations of a single idealized splitting-supercell thunderstorm in order to assess the ability of the algorithm to predict the impacts of MPAR radial velocity observations collected through adaptive, targeted sampling on very short-term convective-scale forecasts of low-level rotation.

Targeted observations for convective-scale data assimilation and forecasts face multiple challenges. Convective-scale processes are predominantly nonlinear, as are the relationships between radar observations (i.e., reflectivity) and model state variables. Using an ensemble-based targeted observation technique on convective scales by assuming linear relationships at different times and between observed variables and forecast metrics presents more obstacles than seen in previous targeted observation studies involving larger scales. Ensemble sampling error on the convective scale can also hinder the ability to appropriately estimate the impacts of observations on subsequent forecasts.

As an initial test of an ensemble-based targeted observation algorithm for convective-scale NWP, a perfectmodel OSSE is designed to negate problems such as model error and uncontrolled observation errors while focusing on ensemble sampling errors and the effects of nonlinearity which have significant impacts on convective scales. Specifically, this study assesses the targeted observation algorithm ability to predict the impact of assimilating synthetic radial velocity observations of a single supercell on a low-level rotation forecast metric (a surrogate for tornado potential) a priori. Three simplified scanning strategies are considered including low-level, midlevel, and high-level to produce various observation subsets of full-volume scans. These three observation sets 

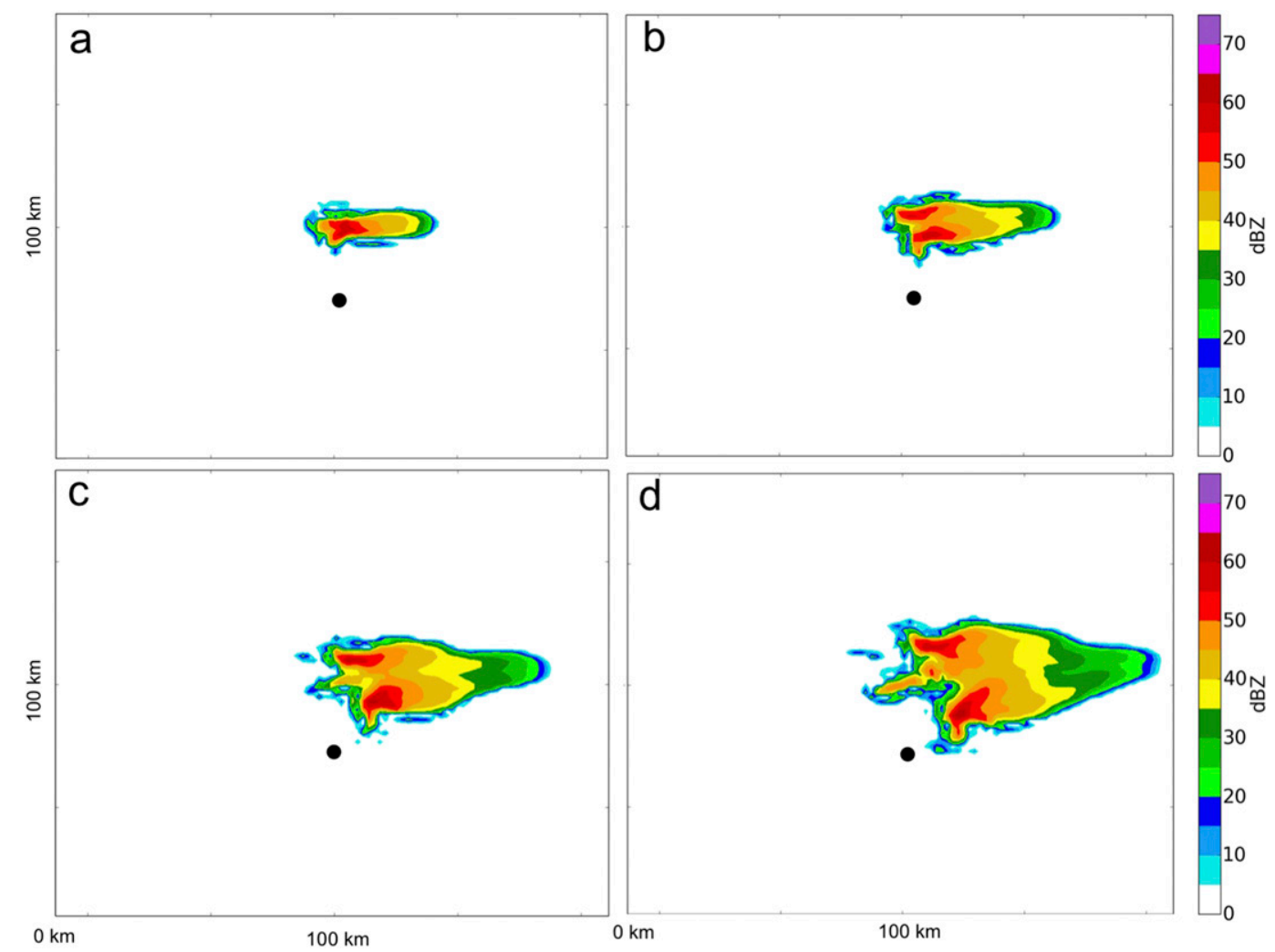

FIG. 1. Truth simulation low-level reflectivity at (a) 45, (b) 60, (c) 75, and (d) 90 min with radar location denoted by a black dot.

will be assimilated independently to reveal the impacts on low-level rotation forecasts compared to full-volume scans. The targeted observation algorithm is also applied to determine whether this technique can appropriately estimate observation impacts on forecasts. Limitations of the algorithm are presented and discussed.

This paper proceeds as follows. Section 2 describes the methodology including ensemble design, targeted observation algorithm, and observations. The analytical results follow in the section 3 . The last section consists of a discussion, conclusions, and broader impacts of the results.

\section{Methodology}

\section{a. Truth simulation and synthetic observations}

Experiments in this study are created using the Advanced Research Weather Research and Forecasting (WRF-ARW, version 3.4.1) Model (Skamarock et al. 2008) initialized with an environment conducive to supercell development. The initial conditions are homogeneous with open lateral boundary conditions. The domain horizontal grid spacing is $2 \mathrm{~km}$ and is $200 \mathrm{~km} \times 200 \mathrm{~km}$. There are 41 vertical levels extending approximately $20 \mathrm{~km}$ with an average $\sim 500 \mathrm{~m}$ spacing dependent on height. The simulations use the Thompson et al. (2008) microphysics scheme with no other parameterizations.

The truth simulation is initialized by a $3-\mathrm{K}$ warm bubble at the center of the domain in an environment supportive of supercell development from both a kinematic and thermodynamic standpoint (Weisman and Klemp 1982). The wind profile is a quarter-circle hodograph with the shear vector turning $45^{\circ}$ over the lowest $2 \mathrm{~km}$. The simulation runs for $2.5 \mathrm{~h}$, creating distinct right- and left-moving supercells (Fig. 1). The rightmover becomes the dominant cell with time (Figs. 1c,d).

Both radial velocity and radar reflectivity observations are harvested from the truth run state (described below) where the assumed Doppler radar is slightly south of the domain center. The storm develops $25 \mathrm{~km}$ north of the radar location. The hypothetical radar sweeps $180^{\circ}$ west-to-east in $5^{\circ}$ azimuth increments over the standard WSR-88D radar 14 elevation angles [volume coverage pattern (VCP 11); Steadham et al. 2002]. All observations are interpolated to the 2-km simulation grid. Radial velocity and reflectivity have observation error variances of $4\left(\mathrm{~m} \mathrm{~s}^{-1}\right)^{2}$ and $4 \mathrm{dBZ}^{2}$, respectively (Yussouf and Stensrud 2010; Sobash and Stensrud 2013; Kerr et al. 2015). The radial velocity forward operator 
consists of the individual wind components along with precipitation fall speeds (Lin et al. 1983) while the reflectivity forward operator requires mixing ratios of rain, snow, and graupel (Ferrier 1994; Gilmore et al. 2004). The synthetic radar observations are created by applying the forward operators to the truth run then applying random noise to account for observation error.

\section{b. OSSE design}

A perfect-model splitting supercell OSSE is conducted. A 50-member ensemble is created by perturbing the homogeneous truth run environment horizontal wind components via uncorrelated Gaussian noise from 0 to $11 \mathrm{~km}$ AGL by $2 \mathrm{~m} \mathrm{~s}^{-1}$. The warm bubble present in the truth simulation is removed from all ensemble members. All observations have a 12-km horizontal and vertical covariance localization (Gaspari and Cohn 1999; Sobash and Stensrud 2013; Kerr et al. 2015). Random perturbations are added to the horizontal wind, temperature, and dewpoint temperature fields at locations where reflectivity observations are greater than $20 \mathrm{dBZ}$, and the reflectivity absolute innovation is greater than $10 \mathrm{dBZ}$ (Dowell and Wicker 2009; Sobash and Wicker 2015). This spins up convection in the appropriate locations and maintains ensemble spread throughout the experiment.

Radial velocity and reflectivity observations are assimilated every 5 min to sufficiently spin up the storm within the ensemble using an ensemble adjustment Kalman filter (EAKF; Anderson 2001) via the Data Assimilation Research Testbed (DART; Anderson et al. 2009). Radial velocity observations collocated with reflectivity values less than $10 \mathrm{dBZ}$ are not assimilated in what is deemed clear air. The assimilation cycling is performed for $60 \mathrm{~min}$ ( 12 cycles) and $75 \mathrm{~min}$ ( 15 cycles) in two independent experiments to spin up convection along with additive noise. Ensemble forecasts are initialized after the repeated assimilation or spinup cycling and are used as backgrounds for targeted observation assimilation and estimation. Reflectivity is only assimilated during this spinup assimilation cycling since radial velocity is the focus of this targeted observation study.

\section{c. Ensemble-based targeted observation algorithm and implementation for radial velocity observations}

The impact of an observation is predicted by estimating forecast metric variance reduction $\boldsymbol{\delta}_{J}$ via:

$$
\boldsymbol{\delta} \boldsymbol{\sigma}_{J^{\prime} t_{2}}=-\left\langle\mathbf{J}_{t_{2}}^{\prime}\left(\mathbf{H x}_{t_{1}}^{\prime}\right)^{\mathrm{T}}\right\rangle\left(\mathbf{H} \mathbf{P}_{t_{1}}^{b} \mathbf{H}^{\mathrm{T}}+\mathbf{R}\right)^{-1}\left\langle\left(\mathbf{H} \mathbf{x}_{t_{1}}^{\prime}\right) \mathbf{J}_{t_{2}}^{\mathrm{T}}\right\rangle
$$

where $\mathbf{J}_{t_{2}}^{\prime}$ is the ensemble perturbation of a scalar metric at forecast time $t_{2}, \mathbf{x}_{t_{1}}^{\prime}$ is the model background ensemble perturbation at the targeted observation time $t_{1}, \mathbf{H}$ is the forward operator that converts the model state to an ensemble estimate of the observation (radial velocity), $\mathbf{R}$ is the observation error covariance, and $\langle *\rangle$ denotes covariance between two quantities. In this study, $\mathbf{H}$ and $\mathbf{R}$ do not vary with time, so the time subscript is omitted. $\mathbf{P}_{t_{1}}^{b}$ is the background ensemble covariance at time $t_{1}$ (Bishop et al. 2001; Ancell and Hakim 2007; Torn and Hakim 2008; Torn and Hakim 2009; Torn 2014). This technique requires no knowledge of the actual observation value thus allows an opportunity to estimate observation impacts before sampling and assimilation. Both simultaneous and serial estimation approaches were proposed previously to estimate the impact of the targeted observations (Bishop et al. 2001; Ancell and Hakim 2007). In this study, the estimation is performed serially, meaning the estimated forecast variance reduction is calculated for each observation individually in sequence, and the total impact of an observation set is determined by a summation of each forecast variance reduction estimate. Since ensemble perturbations are updated after the assimilation of each observation, the ensemble perturbations of $J$ and $\mathbf{H} \mathbf{x}$ also need to be updated before being used by (1) to estimate the error variance reduction for the next observation. Specifically,

$$
\begin{aligned}
\mathbf{H}_{i} \mathbf{x}_{i, t_{1}}^{\prime} & =\mathbf{H}_{i} \mathbf{x}_{i-1, t_{1}}^{\prime}-\mathbf{H}_{i} \tilde{\mathbf{K}}_{i-1}\left[\mathbf{H}_{i-1} \mathbf{x}_{i-1, t_{1}}^{\prime}\right] \\
& =\mathbf{H}_{i} \mathbf{x}_{i-1, t_{1}}^{\prime}-\left(1+\sqrt{\frac{\mathbf{R}_{i-1}}{\mathbf{H}_{i-1} \mathbf{P}_{t_{1}}^{b} \mathbf{H}_{i-1}^{\mathrm{T}}+\mathbf{R}_{i-1}}}\right)^{-1}\left\langle\mathbf{H}_{i} \mathbf{x}_{i-1, t_{1}}^{\prime} \mathbf{x}_{i-1, t_{1}}^{\mathrm{T}} \mathbf{H}_{i-1}^{\mathrm{T}}\right\rangle\left(\mathbf{H}_{i-1} \mathbf{P}_{t_{1}}^{b} \mathbf{H}_{i-1}^{\mathrm{T}}+\mathbf{R}_{i-1}\right)^{-1}\left[\mathbf{H}_{i-1} \mathbf{x}_{i-1, t_{1}}^{\prime}\right]
\end{aligned}
$$

and

$$
\mathbf{J}_{i, t_{2}}^{\prime}=\mathbf{J}_{i-1, t_{2}}^{\prime}-\left(1+\sqrt{\frac{\mathbf{R}_{i-1}}{\mathbf{H}_{i-1} \mathbf{P}_{t_{1}}^{b} \mathbf{H}_{i-1}^{\mathrm{T}}+\mathbf{R}_{i-1}}}\right)^{-1}\left\langle\mathbf{J}_{i-1, t_{2}}^{\prime} \mathbf{x}_{i-1, t_{1}}^{\mathrm{T}^{\prime}} \mathbf{H}_{i-1}^{\mathrm{T}}\right\rangle\left(\mathbf{H}_{i-1} \mathbf{P}_{t_{1}}^{b} \mathbf{H}_{i-1}^{\mathrm{T}}+\mathbf{R}_{i-1}\right)^{-1}\left[\mathbf{H}_{i-1} \mathbf{x}_{i-1, t_{1}}^{\prime}\right],
$$


where $i$ is the $i$ th observation and $i-1$ denotes the observation preceding the $i$ th observation, and $\tilde{\mathbf{K}}_{i-1}$ is the reduced Kalman gain defined as follows:

$$
\begin{aligned}
\tilde{\mathbf{K}}_{i-1}= & \left(1+\sqrt{\frac{\mathbf{R}_{i-1}}{\mathbf{H}_{i-1} \mathbf{P}_{t_{1}}^{b} \mathbf{H}_{i-1}^{\mathrm{T}}+\mathbf{R}_{i-1}}}\right)^{-1} \\
& \times \mathbf{P}_{t_{1}}^{b} \mathbf{H}_{i-1}^{\mathrm{T}}\left(\mathbf{H}_{i-1} \mathbf{P}_{t_{1}}^{b} \mathbf{H}_{i-1}^{\mathrm{T}}+\mathbf{R}_{i-1}\right)^{-1} .
\end{aligned}
$$

$\operatorname{In}(2)-(4), \mathbf{H}_{i-1} \mathbf{P}_{t_{1}}^{b} \mathbf{H}_{i-1}^{\mathrm{T}}=\left\langle\mathbf{H}_{i} \mathbf{x}_{i-1, t_{1}}^{\prime} \mathbf{x}_{i-1, t_{1}}^{\mathrm{T}_{1}} \mathbf{H}_{i-1}^{\mathrm{T}}\right\rangle$ and $\mathbf{P}_{t_{1}}^{b} \mathbf{H}_{i-1}^{\mathrm{T}}=$ $\left\langle\mathbf{x}_{i-1, t_{1}}^{\prime} \mathbf{x}_{i-1, t_{1}}^{T_{1}^{T}} \mathbf{H}_{i-1}^{\mathrm{T}}\right\rangle$. The update of ensemble perturbations in (2)-(4) are required to be consistent with the serial assimilation algorithm.

In (2)-(4), ensemble covariances are used to calculate $\left\langle\mathbf{H}_{i} \mathbf{x}_{i-1, t_{1}}^{\prime} \mathbf{x}_{i-1, t_{1}}^{\prime \mathrm{T}} \mathbf{H}_{i-1}^{\mathrm{T}}\right\rangle$ and $\left\langle\mathbf{J}_{i-1, t_{2}}^{\prime} \mathbf{x}_{i-1, t_{1}}^{\mathrm{T}^{\prime}} \mathbf{H}_{i-1}^{\mathrm{T}}\right\rangle$. Both are subject to the sampling error due to the limited ensemble size. These equations are closely related to the ensemble sensitivity of the forecast metric to the observation ensemble estimate (e.g., Ancell and Hakim 2007; Torn and Hakim 2008):

$$
\frac{\partial J}{\partial(\mathbf{H} \mathbf{x})_{i}}=\left\langle\mathbf{J}_{i, t_{2}}^{\prime} \mathbf{x}_{i, t_{1}}^{\mathrm{T}_{1}^{\prime}} \mathbf{H}_{i}^{\mathrm{T}}\right\rangle\left(\mathbf{H}_{i} \mathbf{P}_{t_{1}}^{b} \mathbf{H}_{i}^{\mathrm{T}}\right)^{-1} .
$$

In this study, the estimated forecast metric variance reduction is only calculated for future observations that are assumed to have an impact on the scalar forecast metric. We adopt the statistical significance test approach to determine if the impact is significant or not. This calculation is a linear regression from which confidence bounds are computed (Wilks 2011, section 6.2.5). A future observation is assumed to impact the forecast metric only if the null hypothesis can be rejected within a specified confidence interval. Large linear regression error results in a significance test failure and observation rejection. For this study, this confidence interval ranges from $95 \%$ to $99 \%$ depending on the point of storm evolution. If an ensemble sensitivity value in (5) does not fit within the confidence bounds, the observation estimate is disregarded and not included in the above workflow. Sensitivity between observation estimates are also calculated to remove observation estimates correlated with other observation estimates. The significance testing of observation correlations does not notably impact the results in this OSSE but is still utilized to be consistent with potential future studies where it may be more impactful. The scalar forecast metric $J$ throughout this study is $0-1-\mathrm{km}$ updraft helicity (hereafter UH01), a metric that quantifies low-level rotation associated with supercells (Kain et al. 2008) while the ensemble estimated observation $\mathbf{H x}$ is radial velocity.

Four observation sets are tested in this study. These include full volume (all 14 elevation angles), low level (elevation angles: $0.5^{\circ}, 1.5^{\circ}, 2.4^{\circ}$ ), midlevel (elevation

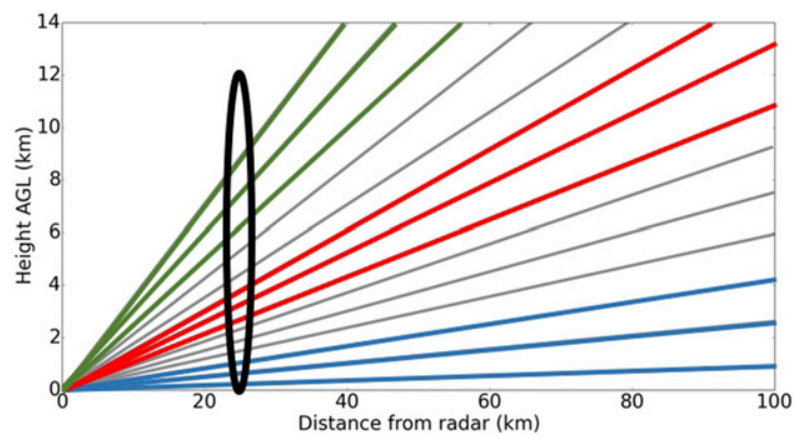

FIG. 2. 14 simulated elevation angles of a full-volume scan with the selected angles for the low level (blue), midlevel (red), and high level (green). The approximate updraft location relative to the radar is illustrated by a black oval.

angles: $6.2^{\circ}, 7.5^{\circ}, 8.7^{\circ}$ ), and high levels (elevation angles: $14.0^{\circ}, 16.7^{\circ}, 19.5^{\circ}$; Fig. 2 ). The ensemble forecasts initialized after storm spinup described above are used as backgrounds for observation assimilation 10, 15, and $20 \mathrm{~min}$ into the forecast windows individually (Fig. 3). The impact of each of the four observation sets is predicted or estimated following (1). Specifically, the targeted observation algorithm is applied to all observation sets using the ensemble forecasts initialized at $t_{0}$ to estimate the impacts on UH01 forecasts at $t_{2}$ via radial velocity observation assimilation at $t_{1}$.

These observation impact predictions are then verified through the actual assimilation of the observation sets. The four observation sets are independently assimilated at each observation time before subsequent forecasts are initialized. Forecast error reduction of UH01 at $t_{2}$ (Fig. 3) is determined by calculating error in forecasts before and after the assimilation. The assimilation impacts are assessed by averaging error over the three $t_{1}$ initialized forecasts to reduce noise attributed to a single forecast. Using a single initialization time and observation set can result in misleading results when comparing various observation set experiments.

\section{d. Targeted observation experiment design and assessment}

In this study, the forecast metric $J$ is defined as UH01 averaged across the domain, or storm, at grid points where UH01 $\geq 5 \mathrm{~m}^{2} \mathrm{~s}^{-2}$. This prevents UH01 not associated with the right-mover mesocyclone from contaminating the results. The left-mover does not have substantial associated low-level rotation.

Since the true state is known in this OSSE, the estimated forecast error variance reduction can be verified using the actual error variance reduction. Error variance is defined as

$$
\text { error variance }=\frac{1}{50} \sum_{n=1}^{50}\left(J_{t}-J_{n}\right)^{2},
$$




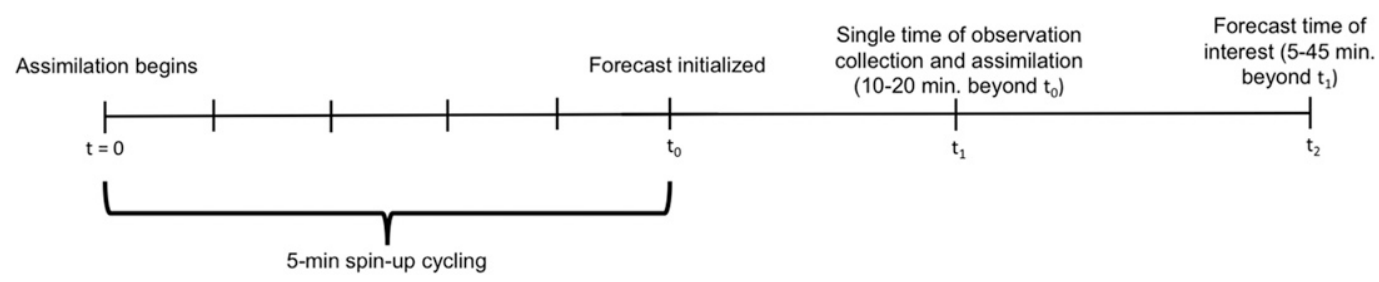

FIG. 3. Schematic for experiments where radial velocity observations are assimilated at $t_{1}$ using an ensemble forecast initialized at $t_{0}$ as the background after repeated spinup assimilation cycling. The impact on UH01 at $t_{2}$ via assimilation at $t_{1}$ is assessed.

where $J_{t}$ is the truth forecast metric and $J_{n}$ is the forecast metric of $n$th ensemble member. The error variance is calculated for the forecasts with and without assimilating the observation sets to obtain the actual error variance reduction or difference. The actual error variance reduction is used to verify the predicted or estimated variance reduction from the targeted observation algorithm (1).

Ensemble forecasts are initialized 60, 75, and $90 \mathrm{~min}$ into the 5-min assimilation cycling procedure described above. These forecasts serve as backgrounds for assimilation of the three observation subsets and fullvolume observation set at various times (10-20 min) beyond the initialization time (Fig. 3). For the 60-min initialization experiments, observations $(70-80 \mathrm{~min})$ are assimilated during the splitting stage of the supercell. The 75-min initialization experiments have observations (85-95 $\mathrm{min})$ poststorm split. Last, the 90-min initialization experiments only have observations at 100 and 105 to show an example when background ensemble variance is smaller. The assimilation impacts are assessed on the basis of reduction in error variance in (6) from the free forecasts at various forecast lead times $\left(t_{2}\right.$; Fig. 3$)$.

\section{Results}

\section{a. Estimate sensitivity to treatment of ensemble sampling errors}

As discussed in section 2c, a statistical significance testing method is used to alleviate the sampling error associated with the limited ensemble size during the error variance reduction estimate (Wilks 2011). Confidence bounds described above are required to only consider observations that have a statistically significant relationship with the forecast metric or that changes in the observation model estimate will induce changes on the forecast metric. The size of this statistical significance confidence interval is critical to ensure accurate estimates of error reduction via observation assimilation. Various confidence intervals are applied individually to sample observation estimates and subsequent forecasts (Fig. 4). The differences are between the estimated and actual error variance reductions as fractions of the background error variance expressed by

$$
\text { Difference }=\frac{(\text { estimated var reduction }- \text { actual var reduction })}{\text { background error var }} .
$$

Therefore, positive (negative) values indicate an over (under) estimation of reduction where zero is a perfect estimate. For an initialized forecast at $60 \mathrm{~min}$ with targeted observations at $75 \mathrm{~min}$ example, a $99 \%$ confidence interval is the closest to zero (Fig. 4a). However, a forecast initialized at $75 \mathrm{~min}$ for targeted observations at $90 \mathrm{~min}$ requires only a $95 \%$ confidence interval to be closest to a perfect forecast (Fig. 4b). This demonstrates the need for an adaptive confidence interval throughout storm evolution. The number of observations that passes the statistical significance test is quite small compared to all observations in each set. At most, only $20 \%$ of available observations are used in the estimate calculations while there are also instances of less than $5 \%$ passing the test, depending on the period of storm evolution and confidence interval value. The linear relationships between radial velocity observations and forecast metrics may be weaker during certain periods of convective storm evolution (e.g., spinup or supercell splitting) compared to periods when storms are mature. These periods of higher nonlinearity would likely require higher confidence intervals. Estimated error variance reductions are verified in the next section at two periods during supercell evolution.

\section{b. Accuracy of the error variance reduction estimation with respect to different storm evolution stages}

As stated above, the linearity between observations and forecast metrics may vary throughout storm evolution due to various processes (supercell splitting in this 

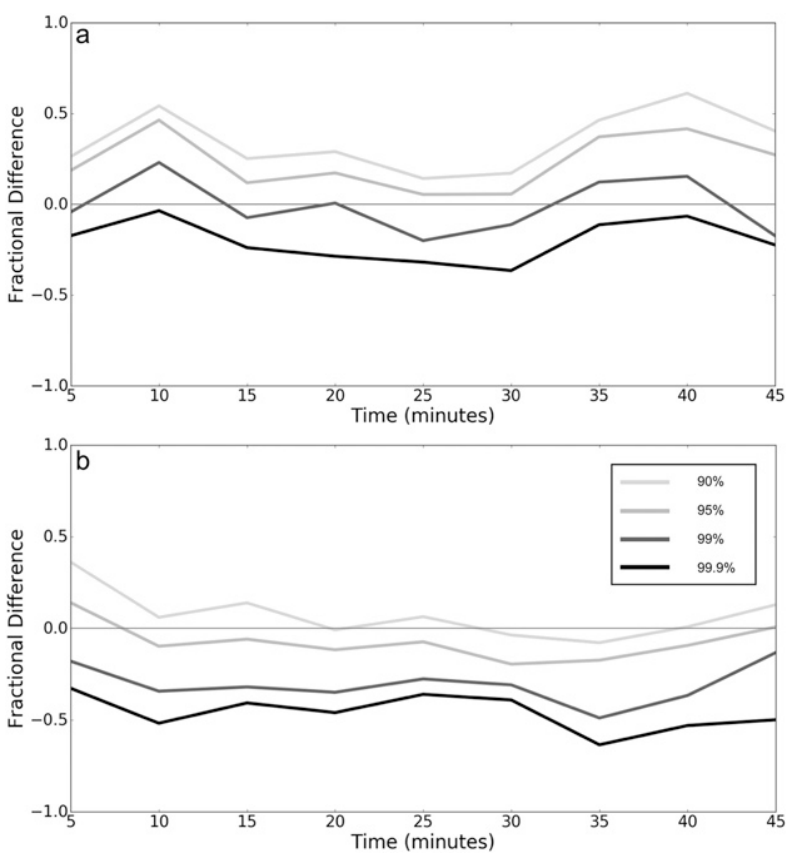

FIG. 4. Normalized estimated variance reduction via assimilation of high-level scan for confidence bounds of $90 \%, 95 \%, 99 \%$, and $99.9 \%$ where positive (negative) values indicate an under (over) estimate for a (a) 60-min initialization example and (b) 75-min initialization example.

case). Therefore, the accuracy of error variance reduction estimations can vary even with adaptive confidence intervals described above, and this method is applied during the storm splitting phase $(60 \mathrm{~min}$; Fig. $2 \mathrm{~b})$ and postsplit/mature phase ( $75 \mathrm{~min}$; Fig. $2 \mathrm{c}$ ). Estimated variance reductions are calculated for 60-min initialization experiments using (1)-(5) (Fig. 5), and the process described in the previous section. Variance reduction estimates for various lead times are shown in general similarly to the actual error variance reductions. The confidence interval used in (5) for 60-min initialization experiments (splitting stage) is $99 \%$. The high threshold reduces effects of sampling errors and nonlinearity prevalent during the splitting stage. One notable overestimate is that of the low-level observation set impact, particularly for a 20- to 30-min lead time. Different from large-scale application, the actual forecast error reductions are noisy throughout the forecast window for all observation set experiments. This creates difficulties for the targeted observation algorithm to predict observation impacts for convective scales.

After the storm split (75-min initialization experiments), forecast variance reduction estimates are again computed but with a confidence interval for (5) of $95 \%$ (Fig. 6). Compared to the results initialized before the storm splitting (Fig. 5), the actual error variance reduction is smoother with respect to the forecast lead times. The variance reduction estimates are more similar in magnitude and lead time behavior as the actual error variance reductions. These results signal a more linear relationship between radial velocity observations and UH01 forecasts after the right-mover has fully split off of the left-mover. Generally, the estimated impacts of each observation set experiment relative to one another are comparable to the actual error variance reductions. The estimate shows a diminished impact of the low-level observation set beyond $15 \mathrm{~min}$ with this set creating the smallest reduction for the remainder of the forecast window. The high-level set reduction estimate

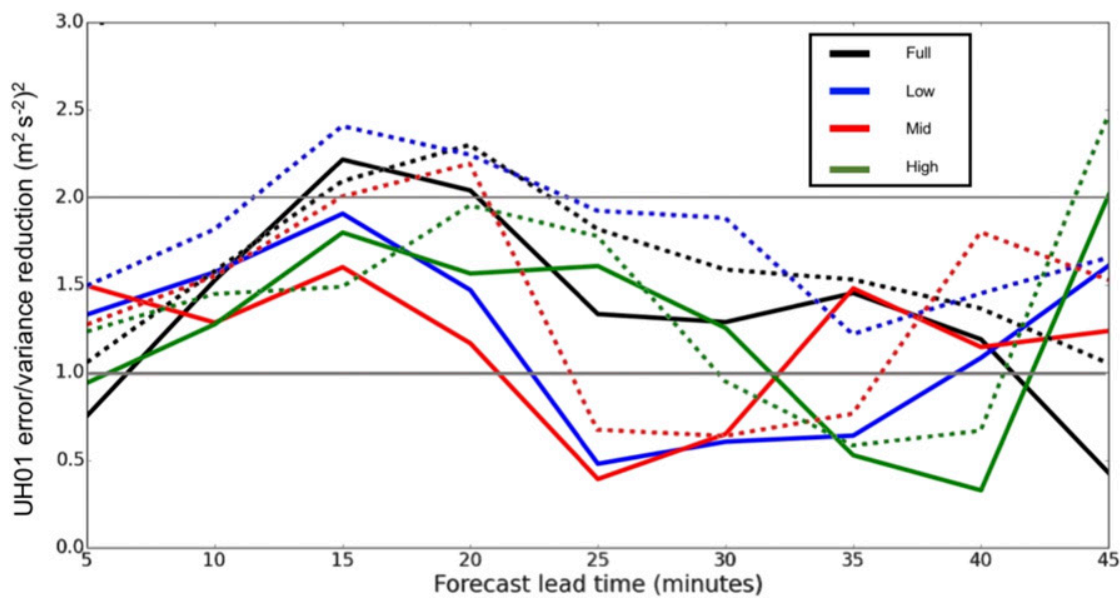

FIG. 5. Full-volume set (black), low-level set (blue), midlevel set (red), and high-level set (green) ensemble variance reduction estimate (dashed) and actual (solid) with forecast lead time averaged over ensemble forecasts initialized after assimilation at 70,75, and $80 \mathrm{~min}$ using a forecast initialized at $60 \mathrm{~min}$. 


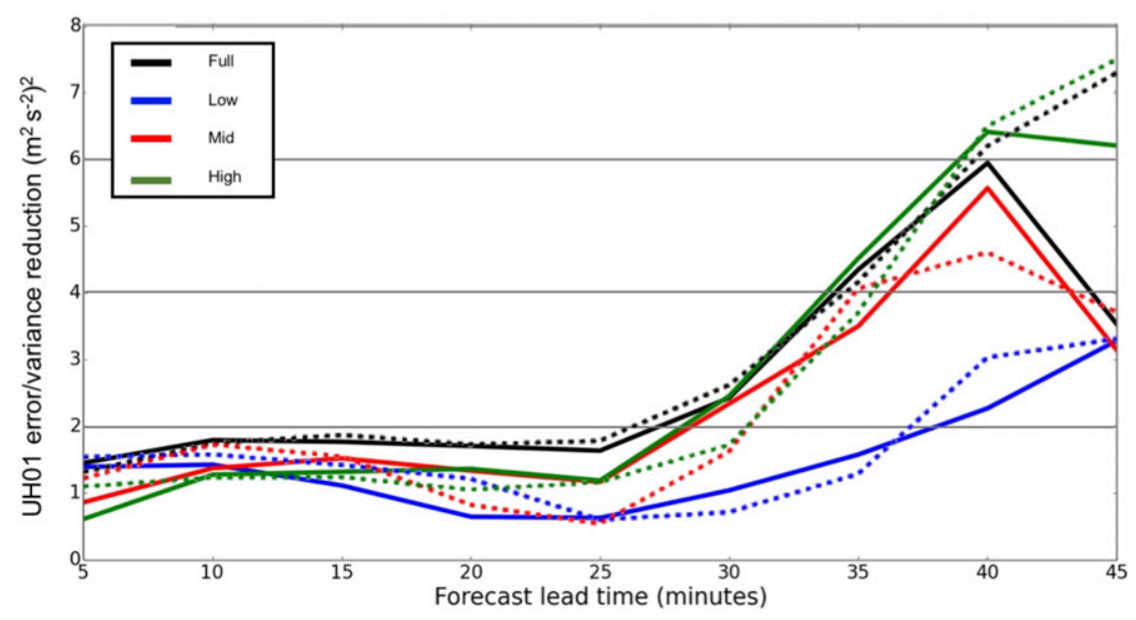

FIG. 6. As in Fig. 5, but for average over ensemble forecasts initialized after assimilation at 85, 90, and $95 \mathrm{~min}$ using a forecast initialized at $75 \mathrm{~min}$.

is not substantially larger than the full-volume set reduction at later lead times (40-45 $\mathrm{min}$ ) as in the actual error variance reductions, but having reduction estimates that are similar should preclude choosing the set with more observations.

\section{c. Impact of adaptive scanning versus full scanning}

Results from the 75-min initialization experiments show the low-level observation set reduces the error variance more than the mid- and high-level sets at $\sim 10$-min lead times (Fig. 6). However, as forecast lead time increases, the low-level set assimilation is less impactful compared to the mid- and high-level sets. By a 35-min lead time, the high-level set has reduced the error variance more than the full-volume scan set (approximately $6 \mathrm{~m}^{2} \mathrm{~s}^{-2}$ at $40 \mathrm{~min}$ ). At this point of storm evolution and radar-relative location, the high sector is sampling the maximum updraft speed height (Fig. 7a). The statistically significant observations for a 30-min forecast determined by (4) for the high sector show a concentration around the right-mover updraft and along the radar-relative updraft peripherals (Fig. 7b). In other words, the updraft vorticity accuracy around the height of maximum speed is crucial for low-level rotation accuracy in the nearfuture (30-45 $\mathrm{min})$.

Comparing example masked correlations between a full-volume assimilation and maximum updraft region assimilation reveals UH01 is affected more by the fullvolume scan in regions that are not dynamically intuitive (Fig. 8a). This includes many noisy correlations within the anvil region. Observations assimilated in locations below the high-sector/max updraft speed region still impact the state within the horizontal cross section given the covariance localization radius. The correlations on the updraft periphery are present on both assimilation experiments and align with the previously described "significant" radial velocity observations (Figs. 7b and 8b), however, the correlations within areas distant from the updraft may have negative impacts on the forecast metric as they are likely
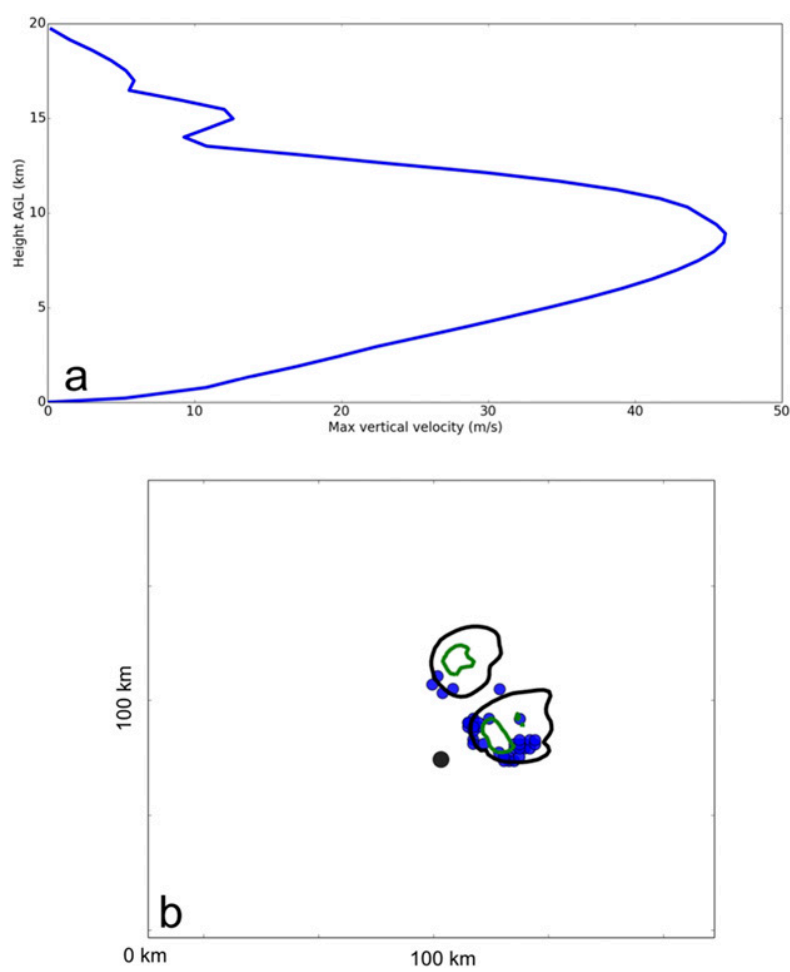

FIG. 7. (a) Truth simulation domain-maximum updraft speed with height at $90 \mathrm{~min}$. (b) "Significant" high-sector scan observations (blue dots) generally along the peripheries of the right-mover updraft $\left(5 \mathrm{~m} \mathrm{~s}^{-1}\right.$, green contour) relative to the radar location (black dot) with reflectivity ( $20 \mathrm{dBZ}$, black contour) at $\sim 11.5 \mathrm{~km}$. 

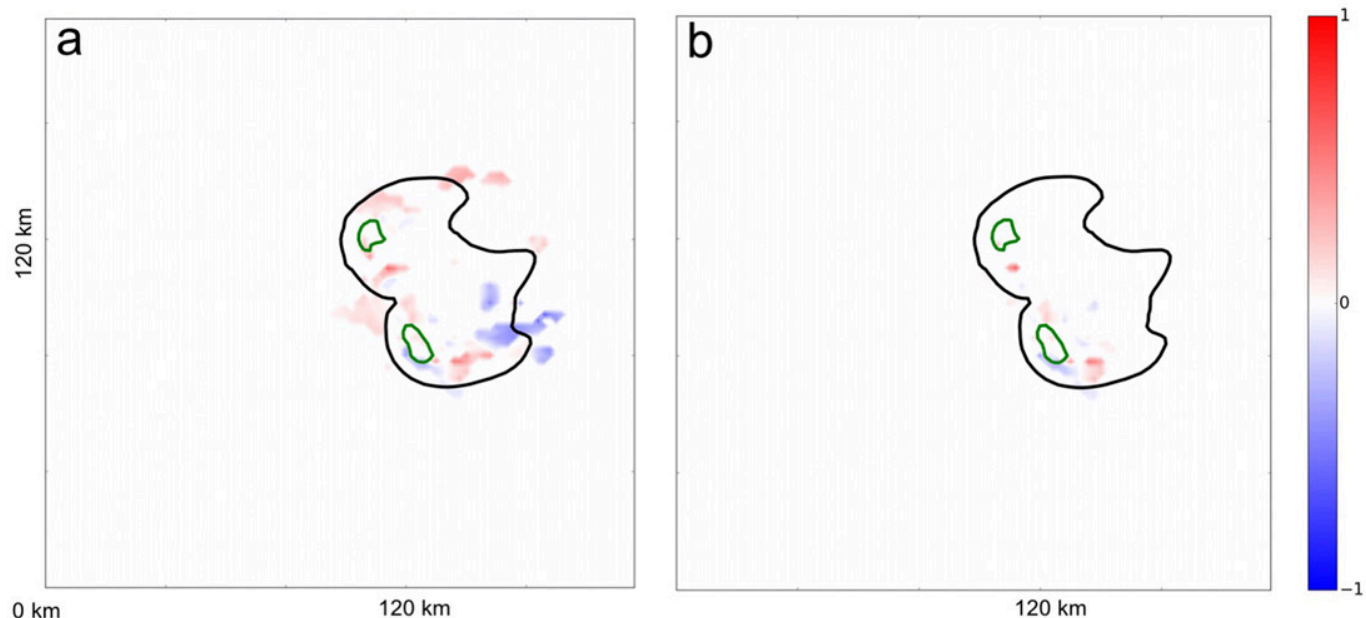

FIG. 8. From the 75 -min initialized forecast, correlations between $\sim 8.5-\mathrm{km}$ horizontal wind speed at $90 \mathrm{~min}$ and 120-min averaged UH01 at locations where the 90-min horizontal wind speed analysis increment is $\geq 2 \mathrm{~m} \mathrm{~s}^{-1}$ after (a) full-volume scan assimilation and (b) high-sector scan assimilation with reflectivity (20 dBZ, black contour) and vertical velocity $\left(5 \mathrm{~m} \mathrm{~s}^{-1}\right.$, green contour).

spurious. Therefore, assimilating all available observations may not necessarily be the best strategy.

\section{d. Overall evaluation of the error variance reduction estimate}

Combing each forecast estimate (108 estimate samples-9 forecast lead times [5-45 $\mathrm{min}$ ] for three observation times, four observation sets) to its respective actual error variance reduction value reveals the general skill of the targeted observation algorithm (1)-(5) for both the 60- and 75-min initialization experiments (Figs. 9a,b). Points below the $45^{\circ}$ slope signify an overestimation of variance reduction while points above the $45^{\circ}$ slope indicate an underestimation for that particular forecast sample. While some individual forecast variance reduction estimates are inaccurate, the overall trend is encouraging as the estimated reductions increase with increasing actual error variance reduction. Normalizing all forecast sample estimates and actual error variance reduction values for both 60 - and 75-min initialization experiments (216 total samples) shows a positive correlation between the predicted impacts and actual, verified impacts via a larger sample size (Fig. 9c).

Following Majumdar et al. (2001), a test to determine whether the estimated variance reduction and actual variance reduction are statistically related. The samples are grouped in three bins of 72 samples based on the lowest, middle, and highest estimated variance reductions (Fig. 9c). The height of each bin is the average actual variance reduction for the bin's samples. Each bin has a $\chi^{2}$ distribution ( 72 degrees of freedom) with a $99 \%$ confidence interval placed on the actual variance reduction. The confidence bounds illustrate it is impossible to show the actual variance reduction as a decreasing function of estimated variance reduction.

\section{e. Accuracy of the error variance reduction estimation with respect to ratio of observation error variance and background ensemble variance}

While the variance reduction estimates are skillful during the development stages, the ability to reasonably estimate variance reduction degrades at later times during storm evolution. An ensemble forecast is initialized at $90 \mathrm{~min}$ after 5 -min assimilation cycling described in the previous section (18 cycles). The impacts of the four observation sets are estimated and verified as in the 60- and 75-min initialization experiments. Through many confidence interval trials and adjustments, the targeted observation algorithm does not provide decent forecast variance reduction estimates, even with a very high observation confidence interval of $99.9 \%$ (Fig. 10a). The regression slope of these samples is not as optimal as the in the earlier time samples (Fig. 9). Over 90 min of assimilation cycling, ensemble spread decreases (not shown), thus the impact of observation assimilation decreases. In other words, the information content in the observation relative to the background forecast is reduced at later data assimilation cycles. The sudden worsening of the variance reduction estimate skill may be due to the smaller overall observation impact on subsequent forecasts.

Model estimated radial velocity variance decreases drastically between the 75- and 90-min initialization experiments (Fig. 10b). For model radial velocity in the 

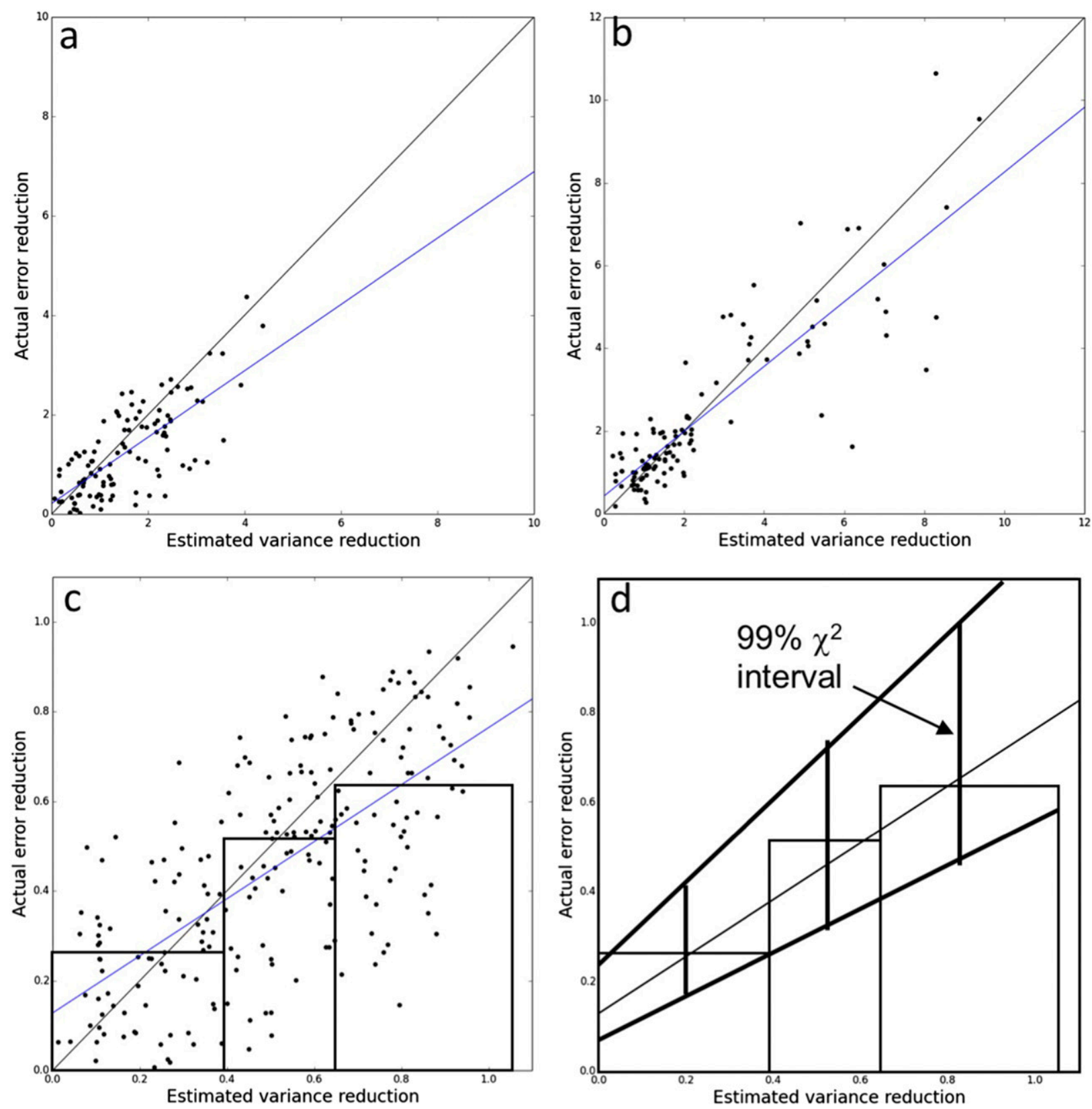

FIG. 9. Scatter of the estimated variance reduction vs actual error variance reduction [both in $\left(\mathrm{m}^{2} \mathrm{~s}^{-2}\right)^{2}$ ] of 45 -min forecasts in $5 \mathrm{~min}$ intervals after assimilating four observation sets at (a) 70,75, and $80 \mathrm{~min}$ with regression slope and $45^{\circ}$ slope (108 samples), (b) 85,90 , and 95 min with regression slope and $45^{\circ}$ slope (108 samples), (c) all times normalized (216 total samples) with three bins for the lowest, middle, and highest estimated variance reduction samples where the height of each bin is the average actual variance reduction with regression slope and $45^{\circ}$ slope, and (d) three bins from (c) with $99 \% \chi^{2}$ interval and bounds with regression slope.

90-min experiments (observations at 100 and $105 \mathrm{~min}$ ), the average $\mathbf{H x}$ less than the radial velocity observation error variance of $4\left(\mathrm{~m} \mathrm{~s}^{-1}\right)^{2}$. The average error of the estimated variance reductions for all lead times for each initialization time are compared to the ratio of radial velocity ensemble variance and observation error variance (Fig. 10c). When ensemble variance is less than observation error variance (ratio $<1$ ), the error is significantly greater than when the ensemble variance is sufficient. Thus, the ensemble becomes too underdispersive for observations to have any meaningful impact on analyses and subsequent forecasts. The targeting algorithm cannot produce accurate impact estimates under these conditions. Therefore, sufficient 

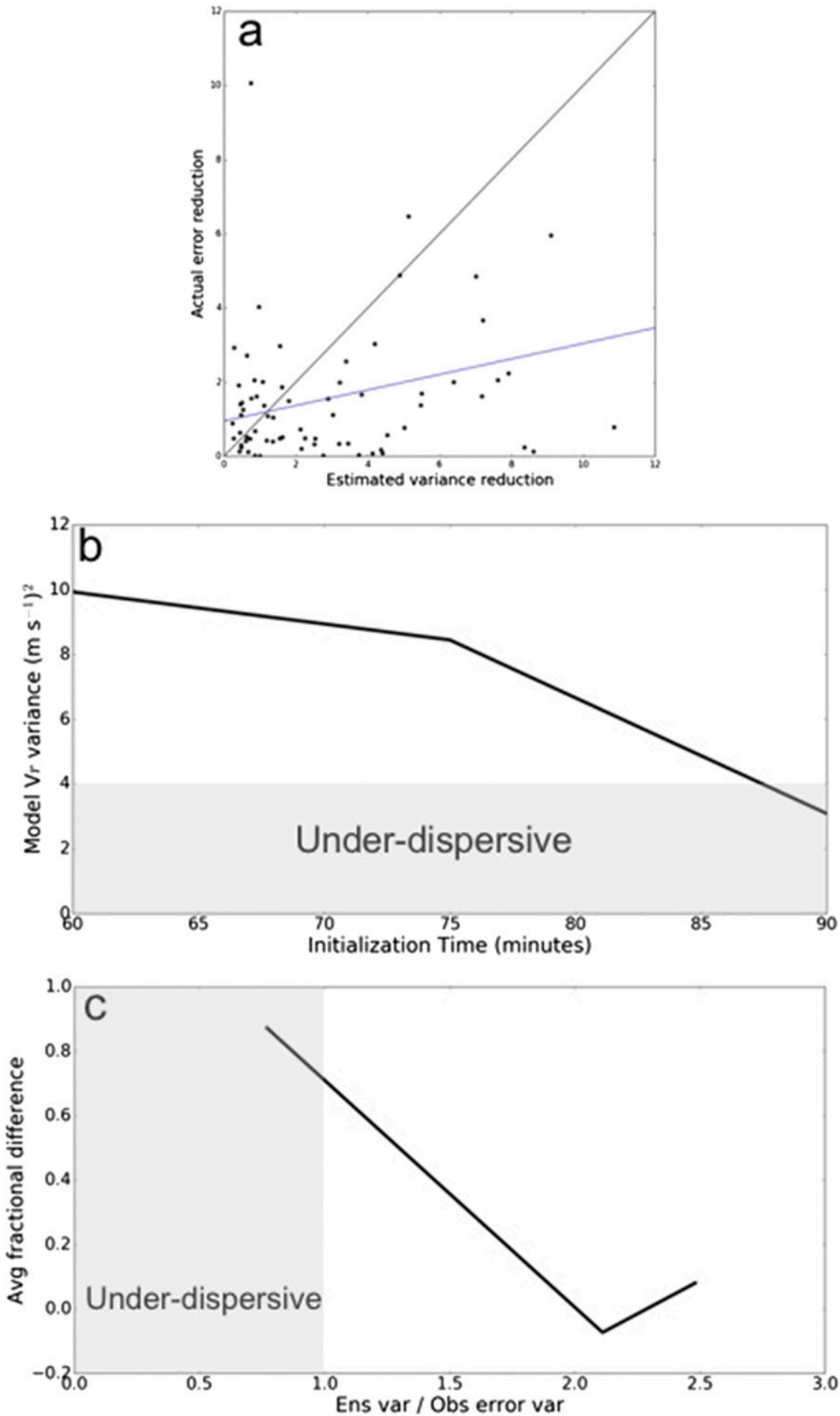

FIG. 10. (a) As in Figs. 9a and 9b, but for observation sets at 100 and 105 min using an ensemble forecast initialized at $90 \mathrm{~min}$. (b) Ensemble variance of model estimated radial velocity observations (full-volume scan average) at various times during storm evolution. (c) Average fractional difference (error) for all lead times of the three initialization times as a function of ensemble variance and observation error variance ratio. 
ensemble spread is necessary for this technique to be suitably utilized.

\section{Discussion and conclusions}

An ensemble-based targeted observation algorithm is applied to Doppler radial velocity observations for the prediction of low-level rotation of a supercell. It is found that when the ensemble has sufficient spread, the ensemble-based estimation can in general reasonably predict the actual error variance reduction. It should be noted that these conclusions are specific to this idealized case, and other cases may yield different results. Lowlevel rotation forecast metrics may be too noisy in less ideal scenarios, forcing the usage of other metrics.

Normalized estimates and actual error variance reductions of the 60- and 75-min forecast initialization times show an encouraging trend and a general correlation between the estimates and actual error reduction (Fig. 9c). This includes the three storm sectors and fullvolume scans. Once ensemble variance of model estimated observations approaches the observation error variance, the ability of the algorithm to accurately predict observation impacts diminishes even with a stricter method to treat sampling errors. This result suggests the targeted radial velocity observations may mostly useful at earlier stage of the data assimilation cycling where large correction associated with large forecast error variance is expected. It is also found that targeted radial velocity observations may be most useful when associated with less nonlinearity. Estimates of forecast variance reduction to lead times beyond $45 \mathrm{~min}$ are poor given sampling error and increased nonlinear relationships between observations and the forecast metric. The accuracy of the estimation of the observation impact tends to be reduced when the forecast period covers a rather nonlinear stage of the storm development (e.g., splitting).

The targeted observation algorithm predicts various sector scans to have comparable or greater impacts on UH01 than full-volume scans at various forecast lead times. The assimilation experiments used to verify the targeted observation predictions show similar impact difference between observation sets. This includes the significance of the maximum updraft speed region for a 30-45-min forecast. The maximum updraft speed region sector observations reduce the forecast variance more than the full-volume scan when assimilated between 85 and $95 \mathrm{~min}$. While the full-volume scan should be assimilated at given times during storm evolution, the maximum updraft speed region may only be necessary at other times. This region is ultimately the dynamical indicator of storm strength and potential longevity. Thus, observations within this zone are crucial to model forecasts. Low-level observations are generally useful for forecast lead times of $15 \mathrm{~min}$ or less. These very short model forecast lead times would likely not be beneficial to meteorologists as are the $30-45$-min model forecasts. Therefore, low-level radial velocity observations may not be important for the intended purpose of an operational WoF ensemble system which is focused on forecast lead times up to $3 \mathrm{~h}$.

A perplexing question that arises from the results is why forecasts sometimes improve by assimilating a subset of the full-volume scan rather than the entire volume. One potential cause of this outcome may be sampling error inducing spurious correlations between some observations within the full-volume scan and UH01. Correlations between the initial condition state horizontal wind speed (a proxy for radial velocity) and 30-min forecast UH01 are masked by horizontal wind speed analysis increments after the assimilation of a fullvolume scan and high-sector (maximum updraft region) scan (Fig. 5). The visible correlations indicate that through the assimilation of radial velocity observations, the horizontal wind speed state is altered thus impacting the ensemble UH01 forecast. A larger ensemble can deter some spurious correlations, but this comes at the cost of increasing the computational expense.

A significant advantage of targeted observations during supercell thunderstorm development would be the opportunity to observe low vertical levels multiple times each minute to benefit human forecasters while only observing the maximum updraft speed region once per minute for potential assimilation. This would provide meteorologists rapidly updating data which has been shown to increase situational awareness and increase warning lead time while also providing the necessary observations for the operational ensemble forecasting system since a subset of a full-volume scan could be sufficient. Reducing the number of observations assimilated significantly also alleviates the computational burden of frequently assimilating radar observations every 1 or 5 min meaning not all observations collected need assimilation.

Future studies should incorporate reflectivity into the targeted observation algorithm. This will be challenging given the high nonlinearity between the model state and observation. Further work can also add a temporal component to the algorithm to estimate the effect of assimilation cycling over short time periods (10-15 min). Also, a study that assesses the impacts of only assimilating observations predicted to have statistically significant impacts on forecast metrics could be intriguing. Other severe convection forecast metrics should be considered such as reflectivity and surface wind speed in 
conjunction with low-level rotation to determine which targeted observations are optimal for all hazards simultaneously. Since the appropriate confidence interval varies with time in this study, further research should consider real-data and imperfect cases to determine suitable confidence interval values when model error can negatively impact the targeted observation algorithm.

Acknowledgments. The authors are greatly appreciative of feedback provided by Aaron Johnson, Mark Weber, Kurt Hondl, Lou Wicker, and Pam Heinselman. Comments by three anonymous reviewers greatly improved the presentation of this study. This study was supported by NA160AR4320115. Some of the results were presented at the AMS Severe Local Storms conference in 2018. The computing for this project was performed at the OU Supercomputing Center for Education and Research (OSCER) at the University of Oklahoma (OU).

\section{REFERENCES}

Aksoy, A., D. Dowell, and C. Snyder, 2009: A multicase comparative assessment of the ensemble Kalman filter for assimilation of radar observations. Part I: Storm-scale analyses. Mon. Wea. Rev., 137, 1805-1824, https://doi.org/ 10.1175/2008MWR2691.1.

Ancell, B., and G. J. Hakim, 2007: Comparing adjoint- and ensemble-sensitivity analysis with applications to observation targeting. Mon. Wea. Rev., 135, 4117-4134, https://doi.org/ 10.1175/2007MWR1904.1.

Anderson, J. L., 2001: An ensemble adjustment filter for data assimilation. Mon. Wea. Rev., 129, 2884-2903, https://doi.org/ 10.1175/1520-0493(2001)129<2884:AEAKFF $>2.0 . C O ; 2$

—, T. Hoar, K. Raeder, H. Liu, N. Collins, R. Torn, and A. Avellano, 2009: The Data Assimilation Research Testbed: A community facility. Bull. Amer. Meteor. Soc., 90, 1283-1296, https://doi.org/10.1175/2009BAMS2618.1.

Baker, N. L., and R. Daley, 2000: Observation and background adjoint sensitivity in the adaptive observation-targeting problem. Quart. J. Roy. Meteor. Soc., 126, 1431-1454, https://doi.org/ 10.1002/qj.49712656511.

Berliner, L. M., Q. Lu, and C. Snyder, 1999: Statistical design for adaptive weather observations. J. Atmos. Sci., 56 , 2536-2552, https://doi.org/10.1175/1520-0469(1999)056<2536: SDFAWO $>2.0 . \mathrm{CO} ; 2$.

Bishop, C. H., B. J. Etherton, and S. J. Majumdar, 2001: Adaptive sampling with the ensemble transform Kalman filter. Part I: Theoretical aspects. Mon. Wea. Rev., 129, 420-436, https://doi.org/ 10.1175/1520-0493(2001)129<0420:ASWTET>2.0.CO;2.

Buizza, R., and A. Montani, 1999: Targeting observations using singular vectors. J. Atmos. Sci., 56, 2965-2985, https://doi.org/ 10.1175/1520-0469(1999)056<2965:TOUSV > 2.0.CO;2.

Chang, W., 2014: Convective-scale radar data assimilation and adaptive radar observation with the ensemble Kalman filter. Ph.D. thesis, McGill University, 154 pp.

Curtis, C. D., and S. M. Torres, 2011: Adaptive range oversampling to achieve faster scanning on the National Weather Radar Testbed Phased-Array Radar. J. Atmos. Oceanic Technol., 28, 1581-1597, https://doi.org/10.1175/JTECH-D-10-05042.1.
Dowell, D. C., and L. J. Wicker, 2009: Additive noise for stormscale ensemble forecasting and data assimilation. J. Atmos. Oceanic Technol., 26, 911-927, https://doi.org/10.1175/ 2008JTECHA1156.1.

, F. Zhang, L. J. Wicker, C. Snyder, and N. A. Crook, 2004: Wind and temperature retrievals in the 17 May 1981 Arcadia, Oklahoma, supercell: Ensemble Kalman filter experiments. Mon. Wea. Rev., 132, 1982-2005, https://doi.org/10.1175/15200493(2004)132<1982:WATRIT>2.0.CO;2.

Ferrier, B. S., 1994: A double-moment multiple-phase four-class bulk ice scheme. Part I: Description. J. Atmos. Sci., 51, 249-280, https://doi.org/10.1175/1520-0469(1994)051<0249:ADMMPF> 2.0.CO;2.

Gaspari, G., and S. E. Cohn, 1999: Construction of correlation functions in two and three dimensions. Quart. J. Roy. Meteor. Soc., 125, 723-757, https://doi.org/10.1002/qj.49712555417.

Gelaro, R., R. Langland, G. D. Rohaly, and T. E. Rosmond, 1999: An assessment of the singular-vector approach to targeted observations using the FASTEX dataset. Quart. J. Roy. Meteor. Soc., 125, 3299-3327, https://doi.org/10.1002/qj.49712556109.

Gilmore, M. S., J. M. Straka, and E. N. Rasmussen, 2004: Precipitation uncertainty due to variations in precipitation particle parameters within a simple microphysics scheme. Mon. Wea. Rev., 132, 2610-2627, https://doi.org/10.1175/MWR2810.1.

Hamill, T., and C. Snyder, 2002: Using improved background-error covariances from an ensemble Kalman filter for adaptive observations. Mon. Wea. Rev., 130, 1552-1572, https://doi.org/ 10.1175/1520-0493(2002)130<1552:UIBECF $>2.0 . \mathrm{CO} ; 2$.

Heinselman, P. L., and S. M. Torres, 2011: High-temporal-resolution capabilities of the National Weather Radar Testbed Phased-Array Radar. J. Appl. Meteor. Climatol., 50, 579593, https://doi.org/10.1175/2010JAMC2588.1.

, D. S. LaDue, and H. Lazrus, 2012: Exploring impacts of rapid-scan radar data on NWS warning decisions. Wea. Forecasting, 27, 1031-1044, https://doi.org/10.1175/WAF-D11-00145.1.

,-- D. M. Kingfield, and R. Hoffman, 2015: Tornado warning decisions using phased-array radar data. Wea. Forecasting, 30, 57-78, https://doi.org/10.1175/WAF-D-14-00042.1.

Johnson, A., X. Wang, J. R. Carley, L. J. Wicker, and C. Karstens, 2015: A comparison of multiscale GSI-based EnKF and 3DVar data assimilation using radar and conventional observations for midlatitude convective-scale precipitation forecasts. Mon. Wea. Rev., 143, 3087-3108, https://doi.org/10.1175/ MWR-D-14-00345.1.

Kain, J. S., and Coauthors, 2008: Some practical considerations for the first generation of operational convection allowing NWP: How much resolution is enough? Wea. Forecasting, 23, 931952, https://doi.org/10.1175/WAF2007106.1.

Kerr, C. A., D. J. Stensrud, and X. Wang, 2015: Assimilation of cloud-top temperature and radar observations of an idealized splitting supercell using an observing system simulation experiment. Mon. Wea. Rev., 143, 1018-1034, https://doi.org/ 10.1175/MWR-D-14-00146.1.

Kuster, C. M., P. L. Heinselman, and M. Austin, 2015: 31 May 2013 El Reno tornadoes: Advantages of rapid-scan phased-array radar data from a warning forecaster's perspective. Wea. Forecasting, 30, 933-956, https://doi.org/10.1175/WAF-D-14-00142.1.

Langland, R. H., and Coauthors, 1999: The North Pacific Experiment (NORPEX-98) Targeted observations for improved weather forecasts. Bull. Amer. Meteor. Soc., 80, 1363-1384, https://doi.org/10.1175/1520-0477(1999)080<1363: TNPENT >2.0.CO;2. 
Limpert, G. L., and A. L. Houston, 2018: Ensemble sensitivity analysis for targeted observations of supercell thunderstorms. Mon. Wea. Rev., 146, 1705-1721, https://doi.org/10.1175/MWRD-17-0029.1.

Lin, Y. L., R. D. Farley, and H. D. Orville, 1983: Bulk parameterization of the snow field in a cloud model. J. Climate Appl. Meteor., 22, 1065-1092, https://doi.org/10.1175/1520-0450(1983) 022<1065:BPOTSF $>2.0$. CO;2.

Maejima, Y., M. Kunii, and T. Miyoshi, 2017: 30-second-update 100-m-mesh data assimilation experiments: a sudden local rain case in Kobe on 11 September 2014. SOLA, 13, 174-180, https://doi.org/10.2151/sola.2017-032.

_- T. Miyoshi, M. Kunii, H. Seko, and K. Sato, 2019: Impact of dense and frequent surface observations on 1-minute-update severe rainstorm prediction: A simulation study. J. Meteor. Soc. Japan, 97, 253-273, https://doi.org/10.2151/jmsj.2019-014.

Majumdar, S. J., C. H. Bishop, B. J. Etherton, I. Szunyogh, and Z. Toth, 2001: Can an ensemble transform Kalman filter predict the reduction in forecast-error variance produced by targeted observations? Quart. J. Roy. Meteor. Soc., 127, 28032820, https://doi.org/10.1002/qj.49712757815.

Palmer, T. N., R. Gelaro, J. Barkmeijer, and R. Buizza, 1998: Singular vectors, metrics, and adaptive observations. J. Atmos. Sci., 55, 633-653, https://doi.org/10.1175/15200469(1998)055<0633:SVMAAO > 2.0.CO;2.

Skamarock, W. C., and Coauthors, 2008: A description of the Advanced Research WRF version 3. NCAR Tech. Note NCAR/ TN-475+STR, 113 pp., https://doi.org/10.5065/D68S4MVH.

Snyder, C., and F. Zhang, 2003: Assimilation of simulated Doppler radar observations with an ensemble Kalman filter. Mon. Wea. Rev., 131, 1663-1677, https://doi.org/10.1175//2555.1.

Sobash, R. A., and D. J. Stensrud, 2013: The impact of covariance localization for radar data on EnKF analyses of a developing MCS: Observing System Simulation Experiments. Mon. Wea. Rev., 141, 3691-3709, https://doi.org/10.1175/ MWR-D-12-00203.1.

— storm-scale EnKF experiments. Mon. Wea. Rev., 143, 30673086, https://doi.org/10.1175/MWR-D-14-00323.1.

Steadham, R. M., R. A. Brown, and V. T. Wood, 2002: Prospects for faster and denser WSR-88D scanning strategies. Preprints, 18th Int. Conf. on IIPS Interactive Symp. on AWIPS, Orlando, FL, Amer. Meteor. Soc., J3.16, https://ams.confex.com/ams/ annual2002/techprogram/paper_25318.htm.

Stensrud, D. J., and Coauthors, 2009: Convective-scale Warn-onForecast system: A vision for 2020. Bull. Amer. Meteor. Soc., 90, 1487-1500, https://doi.org/10.1175/2009BAMS2795.1.

__ , and Coauthors, 2013: Progress and challenges with Warnon-Forecast. Atmos. Res., 123, 2-16, https://doi.org/10.1016/ j.atmosres.2012.04.004.

Stratman, D. R., N. Yussouf, Y. Jung, T. A. Supine, M. Xue, P. S. Skinner, and B. J. Putnam, 2020: Optimal temporal frequency of NSSL phased array radar observations for an experimental Warn-on-Forecast system. Wea. Forecasting, 35, https://doi.org/10.1175/WAF-D-19-0165.1, in press.

Supinie, T. A., N. Yussouf, Y. Jung, M. Xue, J. Cheng, and S. Wang, 2017: Comparison of the analyses and forecasts of a tornadic supercell storm from assimilating phased-array radar and WSR-88D observations. Wea. Forecasting, 32, 1379-1401, https://doi.org/10.1175/WAF-D-16-0159.1.
Thompson, G., P. R. Field, W. R. Hall, and R. M. Rasmussen, 2008: Explicit forecasts of winter precipitation using an improved bulk microphysics scheme. Part II: Implementation of a new snow parameterization. Mon. Wea. Rev., 140, 562-586, https:// doi.org/10.1175/2008MWR2387.1.

Tong, M., and M. Xue, 2005: Ensemble Kalman filter assimilation of Doppler radar data with a compressible nonhydrostatic model: OSS experiments. Mon. Wea. Rev., 133, 1789-1807, https://doi.org/10.1175/MWR2898.1.

Torn, R. D., 2014: The impact of targeted dropwindsonde observations on tropical cyclone intensity forecasts of four weak systems during PREDICT. Mon. Wea. Rev., 142, 2860-2878, https://doi.org/10.1175/MWR-D-13-00284.1.

_- , and G. J. Hakim, 2008: Ensemble-based sensitivity analysis. Mon. Wea. Rev., 136, 663-677, https://doi.org/10.1175/ 2007MWR2132.1.

$\longrightarrow$, and - 2009: Initial condition sensitivity of western Pacific extratropical transitions determined using ensemble-based sensitivity analysis. Mon. Wea. Rev., 137, 3388-3406, https:// doi.org/10.1175/2009MWR2879.1.

Wang, Y., and X. Wang, 2017: Direct assimilation of radar reflectivity without tangent linear and adjoint of the nonlinear observation operator in the GSI-based EnVar system: Methodology and experiment with the 8 May 2003 Oklahoma City tornadic supercell. Mon. Wea. Rev., 145, 1447-1471, https://doi.org/10.1175/MWR-D-16-0231.1.

Weber, M. E., J. Y. Cho, J. S. Herd, J. M. Flavin, W. E. Benner, and G. S. Torok, 2007: The next-generation multimission U.S. surveillance radar network. Bull. Amer. Meteor. Soc., 88, 1739-1752, https://doi.org/10.1175/BAMS-88-11-1739.

Weisman, M. L., and J. B. Klemp, 1982: The dependence of numerically simulated convective storms on vertical wind shear and buoyancy. Mon. Wea. Rev., 110, 504-520, https://doi.org/10.1175/1520-0493(1982)110<0504:TDONSC> 2.0.CO;2.

Wheatley, D. M., N. Yussouf, and D. J. Stensrud, 2014: Ensemble Kalman filter analyses and forecasts of a severe mesoscale convective system using different choices of microphysics schemes. Mon. Wea. Rev., 142, 3243-3263, https://doi.org/ 10.1175/MWR-D-13-00260.1.

Wilks, D. S., 2011: Statistical Methods in the Atmospheric Sciences. 3rd ed. International Geophysics Series, Vol. 100, Academic Press, 704 pp.

Yussouf, N., and D. J. Stensrud, 2010: Impact of phased-array radar observations over a short assimilation period: Observing system simulation experiments using an ensemble Kalman filter. Mon. Wea. Rev., 138, 517-538, https://doi.org/10.1175/ 2009MWR2925.1.

— parameter ensembles for assimilation of radar observations using the ensemble Kalman filter. Mon. Wea. Rev., 140, 562586, https://doi.org/10.1175/MWR-D-10-05074.1.

_ , E. R. Mansell, L. J. Wicker, D. M. Wheatley, and D. J. Stensrud, 2013: The ensemble Kalman filter analyses and forecasts of the 8 May 2003 Oklahoma City tornadic supercell storm using single- and double-moment microphysics schemes. Mon. Wea. Rev., 141, 3388-3412, https://doi.org/10.1175/MWRD-12-00237.1.

Zrnić, D. S., and Coauthors, 2007: Agile-beam phased array radar for weather observations. Bull. Amer. Meteor. Soc., 88, 1753 1766, https://doi.org/10.1175/BAMS-88-11-1753. 